\title{
El Derecho de la Moda, sus principales movimientos y lo más trendy: el Fashion Tech
}

Annalucia Fasson Llosa

Abogada por la Universidad de Lima. Magíster en Finanzas y Derecho Corporativo por la Universidad ESAN. Especialización en Derecho de la Moda por el Fashion Law Institute de

la Universidad Fordham.

Profesora de la Universidad de Lima. Profesora de la Universidad de Ciencias Aplicadas del Perú.

Profesora en la Universidad ESAN.

Profesora en el Instituto Centro de Altos de la Moda. Mentora de emprendimientos de moda y retail de la incubadora Emprende UP de la Universidad del Pacífico.

SUMARI0:

I. Introducción.

II. Principales movimientos en la industria de la moda.

1. Moda sostenible.

2. Fast fashion.

3. Slow fashion o moda lenta.

4. Súper reciclaje o upcycling.

5. Ultra fast fashion.

III. El Fashion Tech.

IV. Conclusiones. 


\section{RESUMEN:}

A través del presente artículo, la autora esboza los principales movimientos del Derecho de la Moda, los cuales se han ido desarrollando a fin de satisfacer las necesidades de los consumidores. Hace hincapié en las tendencias, las cuales no pueden ser delimitadas en la medida que son el producto de nichos específicos de la comunidad.

Palabras clave: Derecho a la Moda, moda rápida, moda lenta, ultra reciclaje, ultra moda rápida, Moda y Tecnología - Innovación.

\footnotetext{
ABSTRACT:

Through this article, the author outlines the main movements of Fashion Law, which have been developed in order to meet the needs of consumers. It emphasizes trends, which cannot be delimited to the extent that they are the product of specific niches in the community.

Keywords: Fashion Law, fast fashion, slow fashion, upcycling, ultra fast fashion, Fashion Tech - Innovation.
}

\section{INTRODUCCIÓN}

En forma de preámbulo al avance de las tendencias en la industria de la moda, es necesario sentar las bases y definir el Derecho de la Moda, con el propósito de esclarecer el concepto ambiguo de la especialización y no limitarlo a lo lindo y glamuroso, ya que el hablar del Derecho de la Moda abarca mucho más.

Partamos de la moda, no solo es ropa, sino que también involucra calzado, joyas -oro, plata y bisutería fina-, perfumes, cosméticos, decoración de interiores, muebles y accesorios en general como carteras, lentes, correas, sombreros, gorros, entre otros.

Gracias a la publicidad nuestro inconsciente ha asociado a la moda como algo banal y superficial, debido a que la industria del marketing nubla nuestra percepción al mostrarnos parcialmente el detrás de cámaras. Exhiben al público un producto lleno de "aspiración" o "belleza", pues la idea es hacer creer que al adquirirlo uno será feliz o se sentirá mejor.

Precisamente en este punto es donde el Derecho entra a tallar, sobre el detrás de cámaras de la moda, es decir, todo el trasfondo que conlleva el lucir una linda prenda o un bonito calzado, las horas-hombres invertidas para crearlas, fabricarlas, comercializarlas y promocionarlas.

Es ahí donde nace la asesoría legal integral al sector del Derecho de la Moda o Fashion Law, especialidad que se encarga de los problemas particulares y características propias del sector que requieren de una intervención legal eficiente.

En nuestro caso particular, Perú no es ajeno a la moda ni al retail, ya que la industria se encuentra ampliamente desarrollada en diversos ámbitos, es el caso del Emporium Gamarra reconocido a nivel Sudamérica o a las empresas textiles que a la fecha fabrican por encargo de grandes marcas de lujo tales como Ralph Lauren o Calvin Klein. Asimismo, cuenta con un nicho de consumidores de las fast fashion, las cuales han logrado destacar en el mercado, y de la otra parte, contamos con un grupo entrañado en la moda sostenible, que sobresale por la fabricación con materiales orgánicos, sin explotar a sus recursos humanos ni al medio ambiente.

Nuestro país necesita desarrollarse y en mi opinión la industria de la moda, textil y el retail en general tiene mucho potencial, pero es necesario que siga un orden determinado. En mi calidad de fashion lawyer, considero que justamente nuestra especialización es entender este negocio y asesorarlo de la manera correcta, ya que miles de peruanos se dedican a esta industria, pero nadie se ha fijado en ella.

Por lo tanto, establezcamos lo que es derecho de la moda, de la mano de una de las pione- 
ras en esta especialidad, Susan Scafidi', esta es una especialización nueva del derecho como:

"(...) Es un campo que abarca la sustancia legal del estilo, incluyendo las cuestiones que puedan surgir a lo largo de la vida de una prenda de vestir, a partir de la idea original del diseñador y continúa todo el camino hasta el armario del consumidor. En términos estructurales, planteo cuatro pilares básicos del Derecho de la Moda: la propiedad intelectual; negocios y finanzas, con subcategorías que van desde la legislación laboral a los contratos sobre bienes inmuebles; el comercio internacional y la regulación gubernamental, las cuestiones relativas a la seguridad y la sustentabilidad; la cultura del consumo y los derechos civiles. Además de su enfoque central en la ropa y los accesorios, el Derecho de la Moda comprende áreas relacionadas, tales como la producción textil, el modelaje, los medios y las industrias de belleza y fragancia (....)".

En virtud de lo anterior, podemos concluir que el Derecho de la Moda consiste en brindar una asesoría legal completa y exponencialmente incisiva a cualquier persona natural o jurídica dedicada al sector de la moda, al sector textil y al sector fashion retail. Derecho de la Moda es la abstracción del canon de negocio, incorporando toda la línea de producción, mediante una asesoría terrenal y realista que incluye diversas especialidades, Derecho Corporativo, real estate, Derecho Tributario, Derecho Laboral, Derecho Financiero, Derecho Regulatorio, Propiedad Intelectual, entre otros. Cabe precisar que esta especialización no es solo Propiedad Intelectual, si bien es un aspecto vital, son distintas ramas que intervienen y que dependen la una de la otra.

\section{PRINCIPALES MOVIMIENTOS EN LA IN- DUSTRIA DE LA MODA}

Tras precisar los conceptos elementales del
Derecho de la Moda, es importante seguirle el paso al desarrollo y a los movimientos en la industria, puesto que todo fashion lawyer debe ser consciente de estas estrategias de venta, su influencia y su desarrollo en Latinoamérica y en el mundo.

\section{Moda sostenible.}

Movimiento que nació en Reino Unido en el año 2007 de la mano de Kate Fletcher y que se preocupa por cumplir con las normas medioambientales y laborales en la industria textil, moda y fashion retail. Lamentablemente, para que esta tendencia se expanda por varios lugares del mundo, en el año 2013 tuvo que salir a la luz gracias a un penoso accidente en Bangladesh, donde tras un derrumbe en las fábricas textiles ubicadas en el Edificio Rana Plaza, murieron más de 1,000 personas que se dedicaban a elaborar prendas para marcas fast fashion y se exhibió al mundo las condiciones deplorables de los trabajadores y que muchos de estos eran niños e inmigrantes, que se veían expuestos a sustancias dañinas para salud y que trabajaban entre 18 a 20 horas al día ganando sólo US\$ 2.00 —Dos y 00/100 Dólares Americanos—, al día.

Es así, que a partir del 24 de abril de 2014 hacia adelante se conmemora el Fashion Revolution Day $^{2}$ rindiendo honores y exigiendo a las empresas manufactureras que contraten a su personal con las condiciones mínimas de trabajo. Gracias a esta triste tragedia, se remeció y se tomó conciencia de los problemas de la industria de la moda. Gran ejemplo de lo mencionado fue el inicio de la elaboración del documental "The True Cost", producida por Andrew Morgan que descubre las verdaderas condiciones de vida de los trabajadores de ese país ${ }^{3}$.

Por lo tanto, se debe entender a la moda sostenible como la tendencia que propone a los compradores el trasfondo del producto que adquie-

1. Susan Scafidi, Derecho y Moda (Buenos Aires: Marcial Pons, 2015), 19-20.

2. Home Fashion Revolution, http://fashionrevolution.org.

3. The True Cost, http://truecostmovie.com. 
ren. Incita a la reflexión detrás un bello vestido o unos elegantes zapatos, busca la preocupación en la forma de producción de las prendas, evalúa los insumos, se preocupa por los trabajadores y sus condiciones y en si abarca todo el detrás de cámaras del sector de la moda.

En nuestro país, para proteger el medio ambiente, se ha establecido la obligación de contar con estudios de impactos ambientales en caso se desee abrir una fábrica o cumplir con los requisitos de comercio justo ${ }^{4}$, es decir, existen organizaciones como el Flocert que posee el sello de Certificación Fairtrade ${ }^{5}$, mediante el cual certifica la calidad y las prácticas justas en la cadena de fabricación y suministro.

Vale mencionar que la sostenibilidad también se encuentra involucrada en el aspecto social, puesto que el movimiento nace principalmente por los abusos laborales cometidos en las fábricas y las condiciones infrahumanas dadas en la tragedia de Bangladesh.

El Derecho siempre está presente, es así que la Declaración Universal de los Derechos Humanos $^{6}$ en el numeral 1 de su artículo 23 señala que: "Toda persona tiene derecho al trabajo, a la libre elección de su trabajo, a condiciones equitativas y satisfactorias de trabajo y a la protección contra el desempleo (...)". También, se opone a la contratación forzada y al trabajo infantil en condiciones abusivas.

Destacable hecho en el Perú fue que en el 2015 se lanzó el primer directorio de la Asociación de Moda Sostenible del Perú-AMSP7, el cual tuvo un gran apogeo e incorpora a la fecha a más de 70 empresas con objetos sostenibles y propuestas a acrecer la moda sostenible en el Perú.
La AMSP clasificó a la moda sostenible en las siguientes categorías:

a) Moda tecnológica: conocida también como moda inteligente, esta consiste en el uso de recursos tecnológicos para beneficiar a los consumidores y al medio ambiente. Este tipo de prendas desarrolladas con el apoyo de la tecnología son denominadas weareable technologies. Como por ejemplo, desde marcas de lujo como "Hugo Boss" con el desarrollo de calzado sostenible mediante la innovadora alternativa de cuero hecho de las fibras de la hoja de piña "Piñatex" hasta la fast fashion con la gigante "H\&M", mediante su incorporación de la espuma flexible hecha a base de plantas que utiliza la biomasa de algas denominada "Bloom Foom" y que está incorporada dentro de su línea "Conscious Exclusive".

b) Moda ecológica: rama que difunde la protección al medio ambiente, y que busca sintonizar el sistema ecológico con el sistema económico. En otros términos, se ha propuesto el desarrollo económico sin anteponerlo ante los recursos naturales, sino protegiéndolo e innovando en materiales o reciclando, como por ejemplo el vestido de Emma Watson que lució en el Met Gala de 2016 hecho de botellas de plástico.

c) Moda étnica: corriente que se encarga de revalorizar, recuperar y/o promover las técnicas, procedimientos y diseños ancestrales o de comunidades autóctonas de un determinado país, como por ejemplo la marca peruana "Las Polleras de Agus" que trabaja conjuntamente con las comunidades andinas.

4. Coordinadora Nacional del Comercio, http://comerciojusto.pe.

5. FLOCERT apoya las empresas sostenibles y soporta el comercio justo, http://www.flocert.net/es.

6. Declaración Universal de Derechos Humanos, http://www.un.org/es/universal-declaration-human-rights.

7. Asociación de Moda Sostenible del Perú, http://www.amsperu.org. 
Cabe destacar que es importante diferenciar dentro de este movimiento los conceptos de apropiación cultural e inspiración cultural, siendo este primero ajeno a la moda étnica pues se da cuando alguien extrae un elemento cultural perteneciente a un grupo social o comunidad y lo utiliza en su provecho -económico- borrando su origen o relativizándolo, como por ejemplo el famoso caso entre la marca Kuna vs. la comunidad shipiba o el de la diseñadora Isabel Marant vs. la comunidad mixe en Santa María, Tlahuitoltepec, México, mientras la moda étnica si cuenta con el segundo, ya que la inspiración es explícita y ocurre en aquellos casos en que el diseñador o la firma de moda de turno alude claramente al origen de sus diseños.

d) Moda ética: valora principalmente el respeto de los derechos humanos y laborales de sus trabajadores, teniendo como preocupación que todos los trabajadores de la industria textil, moda y fashion retail obtengan un desarrollo profesional y crezcan como personas.

Dentro de la moda ética se están constituyendo empresas de este sector con propósito social o las conocidas como empresas de triple impacto que rescatan no solo la parte de utilidades, sino también el aspecto social y el aspecto medio ambiental. Por ello, a la fecha existen empresas certificadoras que avalan el cumplimiento no solo de las normas laborales o medioambientales sino del buen gobierno corporativo.

De hecho, el viernes 23 de octubre de 2020 se publicó en el Congreso de Perú el Proyecto de Ley 2533-2017-CR, Ley de la Sociedad de Beneficio e Interés Colectivo (Sociedad $\mathrm{BIC})^{8}$, el mismo que entre otras consideraciones destaca lo siguiente: (i) a la denominación social de la persona jurídica societaria se le agregará la expresión "de beneficio e interés colectivo" o la sigla "BIC" y su objeto social puede ser social y/o ambiental y deberá incluir las actividades que colaborarán para la realización de dicho objeto; (ii) en caso de sociedades existentes, se deberá modificar el estatuto, debiendo concurrir en primera convocatoria, cuando menos la concurrencia de dos tercios de las acciones suscritas con derecho a voto y en segunda convocatoria, cuando menos las tres quintas partes de las acciones suscritas con derecho a voto o la mayoría calificada según lo requiera cada tipo societario, el accionista o socio que no esté de acuerdo puede manifestar su derecho de separación; (iii) los directores o administradores, según el tipo de sociedad, velarán por el real cumplimiento del propósito de beneficio social y ambiental e introducirán prácticas de transparencia en sus organizaciones, así como encargarán a un tercero independiente para que elabore un Informe de Gestión sobre las actividades que conlleven al propósito social y/o ambiental establecido en el estatuto, que se publicarán conjuntamente con los resultados económicos de la empresa del ejercicio anterior, en el portal de la sociedad $\mathrm{u}$ otro medio electrónico de comunicación similar utilizado por esta y; (iv) en caso la sociedad incurra en un acto de publicidad engañosa o de competencia desleal, el Instituto Nacional de Defensa de la Competencia y de la Protección de la Propiedad Intelectual-INDECOPI lo sancionará (greenwashing) .

\section{Fast fashion.}

Tal como existe la comida fast food o comida rápida, en la industria de la moda existe el fast fashion, esta consiste en la producción en serie

8. A la fecha de redacción de este artículo no se ha publicado este Proyecto de Ley en el diario oficial El Peruano.

9. Se conoce como greenwashing a la práctica que realizan algunas empresas, vendiéndose como empresas ecológicas o medioambientales — marcas verdes — sin realmente serlo. 
y de forma veloz mediante la cual se lanza entre 15 a 20 colecciones anuales, con la característica esencial de rotar frecuentemente sus productos a costas de una calidad mediana o poca de la ropa.

Asimismo, estas colecciones se venden a bajo precio o low cost. Los productos siguen las últimas tendencias de la moda, pero cuentan con la virtud del factor low cost que les permite el logro de dos finalidades: lograr moda accesible y que las colecciones sean exhibidas por pocas semanas. Es así, que este movimiento busca la alta rotación de inventario y por ende más consumo, logrando mayores ventas y adicción a las compras.

Existió una gran disputa en la cual el fast fashion era tildado como productores de copias o imitaciones de diseñadores exitosos y de grandes pasarelas, mediante las cuales producen los productos a precios menores al original y con menor calidad, pero que cumplen con satisfacer las necesidades rápidas del mercado.

Sin embargo, a la fecha se ha desmentido, gracias a la presencia de marcas internacionales como Zara y H\&M, que cuentan con procesos creativos, innovadores y diferenciadores que permite evadir a la copia, mediante sus propios diseñadores que se encargan de una reinterpretación del modelo original.

Ahora bien, en la actual coyuntura en la que estamos viviendo a raíz de la pandemia del COVID-19, es probable que este movimiento se reinvente y se redefina, toda vez que muchas empresas han dejado de lanzar colecciones, así como no se han presentado a desfile, aun cuando algunos de ellos lo han realizado de manera virtual. Todo hace pensar que el movimiento de fast fashion tal y como lo conocemos mutará. Habrá que esperar un poco para saber cómo se reformula este movimiento el próximo año.

\section{Slow fashion o moda lenta.}

Tendencia que surge como contrapartida al fast fashion. Así como existe el fast food o comida rápida y como contraposición existe el slow food o comida lenta, de igual manera existe el slow fashion que promueve el lanzar una sola colección al año, clásica y atemporal, en otras palabras, "No Season", sin seguir el estresado desarrollo y constante cambio de la rotación de colecciones.

El slow fashion se enfoca en el desarrollo de modelos exclusivos, que se caracterizan por tener costos más elevados, ya que las marcas integrantes del movimiento se basan en la calidad y las materias primas de los productos. Caso contrario al fast fashion, aprecian más la calidad que la cantidad y buscan que perduren al pasar de los años y generen un consumo consciente.

Es así que los consumidores no adquieren las prendas por los diseños llenos de glamour, sino que apoyan el motivo de los productores y son conscientes de los valores y materiales de estas. Uno de los exponentes de este movimiento es la marca Slowear ${ }^{10}$, siendo uno de los principales lemas de este movimiento el que señala que: "Estará largo tiempo a tu lado".

\section{Súper reciclaje o upcycling.}

Se usa mucho en las joyas decoración y en la ropa vintage. Consiste en reciclar elementos antiguos o en desuso y transformarlos otorgándoles una segunda utilidad o uso. Por ejemplo, poniéndole a un terno una capucha o reuniendo varias piezas, como utilizar plumas para crear un collar.

Es como darle una segunda vida al crear un nuevo producto, el mismo que se vende a valor de mercado como un producto nuevo y final.

\section{Ultra fast fashion.}

Consiste en acortar sus procesos de produc-

10. Slowear, http://www.slowear.com/es. 
ción, tal es así que, si una fast fashion como Zara o H\&M se demoran 6 semanas en promedio en fabricar una prenda, marcas como Boohoo"1, Asos $^{12}$ o Missguided se demoran dos o una semana en comercializar sus productos, sin tener problemas de logística, es decir, no tienen problemas en la entrega en el plazo ofrecido y cuentan con un inventario de productos. Cabe precisar, que a raíz de la pandemia COVID-19 este movimiento ha ganado muchos adeptos y muchas marcas están vendiendo bajo esta modalidad, pero dándole énfasis a la categoría de ropa cómoda, tales como buzos, joggers, zapatillas, entre otros.

\section{EL FASHION TECH}

Junto a las nuevas tendencias surgen los nuevos negocios, por eso es importante tomar en cuenta que sucesivamente los emprendedores cuentan con más oportunidades para la creación y desarrollo de un negocio con el uso de la tecnología. Es así como surge el concepto de Fashion Tech, un término que va de la mano con la moda tecnológica anteriormente mencionada y que significa la unión del sector moda, textil o retail con la tecnología.

A continuación, los ejemplos más destacables de la categoría:

a) Novozymes: procedimiento que consiste en la técnica de los jeans deslavados, que parece que se les hubiera echado lejía.

b) Tela Spandex: tela elástica que se utiliza mucho para las ropas de baño y que a la fecha es mundialmente conocida como Lycra.

c) Polos tecnológicos: nuevos productos que Nike y Adidas han presentado como los polos anti-rayos ultravioletas, que no permiten que los rayos solares penetren la tela y lleguen a la piel, así como también polos anti-sudoración, entre otros. d) El uso de la impresora 3D: mediante un software se puede imprimir la tela y establecer las medidas que se desea para literalmente confeccionar una prenda "a la medida".

e) Elektrocouture: consiste en la incorporación de los leds en las prendas, como lo vimos, por ejemplo, en el Met Gala denominado "Manus x Machina: Fashion in the Age of Technology", cuando la modelo Karolina Kurková lució un vestido que cambiaba de colores según las emociones que se recibía de tweets del público o en el Berlin Fashion Week del año pasado.

f) Weareable technologies: es el caso de sacos solares que sirven como batería para recargar celulares.

g) Apps de moda: la creación de aplicaciones relacionadas al sector de la moda, como por ejemplo, la aplicación "Chicisimo App Moda y Ropa", la cual descargas y te permite ver no solo looks sino también dónde comprar las prendas y accesorios, entre otros.

h) Syma Lime: tecnología que consiste en insertar música en la ropa, se le conoce como "visualización del sonido".

En fin, las startups consisten en las empresas nuevas que presentan una fórmula de negocio innovadora y asociada a la tecnología, pero que tienen como prioridad el desarrollo de ideas creativas y diferenciadores del común de mercado. Buscan ser los pioneros y el posicionamiento en el mercado.

En ese sentido, el surgimiento de nuevas ideas creativas conlleva el nacimiento de startups y que, al incorporar tecnología, son parte de la tendencia Fashiontech o Fashtech.

En el Perú cada vez es mayor el interés en el desarrollo de productos innovadores y nuevas

\footnotetext{
11. Boohoo, http://es.boohoo.com/.

12. Asos, http://www.asos.com/.
} 
tecnologías, es así que existen tanto organizaciones privadas como gubernamentales que promueven el surgimiento de estas, siendo las más destacadas "Wayra Perú" en el sector de emprendimiento digital y "StartUp Perú", la iniciativa gubernamental que apoya los productos y servicios innovadores.

En consecuencia, las ideas innovadoras, necesitan contar con los medios suficientes para poder ser plasmados como negocios en el mercado, por ende, será necesario contar con un capital semilla - seed stage - como financiamiento inicial para la creación del modelo de negocio o para permitir el arranque y/o consolidación de la actividad empresarial, debido a que en un primer momento será necesario introducir el modelo al público y no necesariamente será la favorita. Sin embargo, paulatinamente se podrá alcanzar una respuesta favorable.

Posteriormente, y una vez superada la primera etapa, es un factor común la necesidad de apalancar el negocio en busca de crecer y desarrollarse. Es así que en el Perú los emprendedores tecnológicos pueden conseguir recursos a través del Fondo Marco para la Innovación, Ciencia y Tecnología —FOMITEC — o del Fondo de Investigación y Desarrollo para la Competitividad -FIDECOM-. También pueden participar en los concursos que convoca el Ministerio de la Producción en www.start-up.pe.

Asimismo, existen otras modalidades de financiamiento como las siguientes:

a) Crowdfunding: es un mecanismo por el cual el emprendedor presenta un modelo de negocio y el financiamiento requerido para su desarrollo. La idea del proyecto empresarial se publicará en medios virtuales por un plazo de 30 a 90 días, en donde se somete a evaluación y se decide la apro- bación o no del modelo. En el Perú, existen ejemplos como "Creafunding"13, "Afluenta"14, entre otras.

b) Business angels: o inversores ángeles ${ }^{15}$, consiste en un programa de financiamiento, mediante la cual inversionistas analizan los diversos proyectos y eligen los de mayores posibilidades de ingresos, de esta manera deciden financiarlos bajo su propio riesgo. Asimismo, en su calidad de inversionistas también comparten su experiencia, conocimiento y contactos, a través de lo que se le conoce como mentoring.

c) Organismos No Gubernamentales-ONG: son organizaciones orientadas a brindar apoyo económico a diversos emprendimientos enfocados en la responsabilidad social y el desarrollo sostenible. Como proyectos que buscan resguardar el medio ambiente o el desarrollo de comunidades, un claro ejemplo del mecanismo es la ONG Minkando, que consiste en una organización formada por jóvenes que viene promoviendo el desarrollo sostenible por medio de diversos proyectos.

Específicamente, en la industria de la moda existen fondos de inversión denominados Fashion Capital Partners, que son empresas dedicadas a invertir en empresas tecnológicas de moda y lujo, así como en diseñadores innovadores en toda Europa y en los Estados Unidos. Se les conoce en el Reino Unido como Fashion Angels y en Estados Unidos como Pipeline Angels.

En el caso del Perú, contamos con diversas incubadoras o aceleradoras tales como Emprende UP de la Universidad del Pacífico, la Startup de la Universidad Peruana de Ciencias Aplicadas - UPC_, Fab Lab ESAN o la UTEC Ventures como aceleradora de proyectos de empren-
13. https://www.creafunding.com/
14. https://www.afluenta.pe
15. http://angelventures.vc/ 
dimiento. Ahora bien, si hablamos específicamente de moda podemos mencionar que en España ha nacido una incubadora que facilita el surgimiento de emprendimientos específicos de moda y textil que se llama Atelier by ISEM Fashion Business School y que forma parte de la escuela de negocios de la Universidad de Navarra, la cual pretende apostar por darles una oportunidad para que posicionen su marca no sólo en España sino también a nivel internacional.

\section{CONCLUSIONES}

Hemos podido mostrar los diferentes movimientos que existen para satisfacer las necesidades y estilos de vida de sus consumidores, reflejando los múltiples cambios y modalidades tanto de valores como de sus comportamientos. No es preciso delimitar si estas tendencias son buenas o malas, sino que se debe entender que individualmente presentan sus particulari- dades y son producto de la respuesta a nichos específicos de la comunidad, ya que todas estas tendencias recaen sobre el punto común de reforzar la relación entre los productos y sus consumidores, sea por la forma en como los ofrecen o generando o no necesidades en los productos que sean vinculables a los distintos valores o estilos de vida de las personas.

Sin embargo, a raíz de la pandemia del COVID-19, es una coyuntura complicada para el sector textil, moda y fashion retail, dado que se encuentra fuertemente golpeada. Sin embargo, se ha visto un crecimiento exponencial del movimiento de moda sostenible, por lo que la sostenibilidad más que una tendencia ya es una realidad para muchos negocios de este sector.

Asimismo, el sector se está transformando y usará la tecnología en los materiales de las prendas de vestir y accesorios en general para

Nacho Espada. «El modelo 'start up' en la empresa de moda», El Altavoz, 10 de febrero de 2017, https://www.modaes.com/blogs/elaltavoz/el-modelo-start-up-en-la-empresa-de-moda.html.

«El potencial de la Fashion Tech», Euronews, http://es.euronews.com/2017/01/27/el-potencial-de-la-fashion-tech.

Gabriel Farias Iribarren, «La era del Ultra-Fast Fashion», MEXCostura, 6 de octubre de 2017, http://www.mexcostura.mx/noticias/moda/1035-la-era-del-ultra-fast-fashion.

Gabriel Farias Iribarren, «Del Fast Fashion al Ultra Fast Fashion», Gabriel Farias Iribarren, 7 de julio de 2017, https:// gabrielfariasiribarren.com/del-fast-fashion-al-ultra-fast-fashion/.

Annalucia Fasson Llosa, «El Derecho de la Moda y el detrás de cámara de los movimientos Fast Fashion, Slow Fashion, Moda Sostenible y Upcycling», Conexión ESAN, 28 de septiembre de 2015, https://www.esan.edu.pe/ conexion/actualidad/2015/09/28/derecho-moda-detras-camara-movimientos-fast-fashion-slow-fashion-moda-sosten/.

Annalucia Fasson Llosa, «El Derecho de la Moda y los financiamientos que están dando la hora», LexLatin, 3 de agosto de 2016, https://lexlatin.com/opinion/derecho-la-moda-las-formas-financiamiento-estan-dando-la-hora.

Annalucia Fasson Llosa, «Moda 2.0: Cuando la tecnología y el glamour se unen para fortalecer una industria», Conexión ESAN, 28 de agosto de 2017, https://www.esan.edu.pe/conexion/actualidad/2017/08/28/moda-20-cuando-la-tecnologia-y-el-glamour-se-unen-para-fortalecer-una-industria/.

Annalucia Fasson Llosa, «El empoderamiento del Fashion Law como influencer en la economía», Conexión ESAN, 26 de febrero de 2018, https://www.esan.edu.pe/conexion/actualidad/2018/02/26/la-industria-de-la-moda-influencer-en-la-economia-y-el-derecho/.

Annalucia Fasson Llosa, «La última revolución de la moda se llama ultra fast fashion», Conexión ESAN, 24 de mayo de 2018, https://www.esan.edu.pe/conexion/actualidad/2018/05/24/la-ultima-revolucion-de-la-moda-se-llama-ultra-fast-fashion/\#: :text=Las\%20marcas\%20ultra\%20fast\%20fashion,Per\%C3\%BA\%20para\%20revolucionar\%20el\%20mercado. 
generar un impacto positivo en la sociedad, por lo que todo hace pensar que el fashion tech será una alternativa que escogerán muchas empresas del sector para reinventarse, es decir, ya no solo se venderá glamour o lujo, sino que la conexión con el consumidor será más funcional mostrando a la moda como una industria que le permita mejorar su calidad de vida.

En conclusión, esperemos que nuestras autoridades y la comunidad peruana tomen consciencia sobre el sector moda, que se generen nuevas leyes de fomento e incentivo no solo a los sectores tradicionales como los clásicos sectores agrario, pesquero o minero; puesto que, la moda y retail es pasada de largo cuando en realidad ya viene cumpliendo un rol protagónico en nuestro país, Latinoamérica y el mundo. La respuesta ideal del gobierno sería una normativa que no obstaculice, sino que más bien facilite y los promueva, así como también otorgue beneficios tributarios a los emprendedores y empresarios que se dedican a esta industria para fomentar su desarrollo, lo cual haría que no solo crezca en nuestro país, sino que nuestras marcas compitan a nivel internacional como es en otros países de Latinoamérica, generando más ingresos en beneficio de todos los peruanos.

Annalucia Fasson Llosa, «Fashion Tech, innovación, tecnología y moda», Gestión, 20 de mayo de 2018, https:// gestion.pe/opinion/fashion-tech-innovacion-tecnologia-moda-233953-noticia/.

Annalucia Fasson Llosa, «Las tendencias del retail para el año 2019», Idealex, 10 de enero de 2019, https://idealex. press/retail-tendencias-para-el-ano-2019/.

«Fast Fashion Speeding Toward Ultrafast Fashion», Fund Global Retail \& Technology, https://www.fungglobalretailtech.com/wp-content/uploads/2017/05/Fast-Fashion-Speeding-Toward-Ultrafast-Fashion-May-19_2017-DF.pdf.

Vivian Hendriksz, «Boohoo, Asos \& Missguided pave the way for 'Ultrafast Fashion'», Fashion United, 24 de mayo de 2017, https://fashionunited.com/news/fashion/boohoo-asos-missguided-pave-the-way-for-ultrafast-fashion/2017052415984.

«Fashion Tech Women, un evento protagonizado por mujeres que lideran el sector de la moda», Madrid Capital de Moda, http://www.madridcapitaldemoda.com/noticias/fashion-tech-women/.

Natura. Sobre Nosotros, http://www.natura.com.pe/natura/acerca-de-natura/sobre-nosotros.

«Colaborativo y digital el futuro de la moda», Piloto 151, https://piloto151.com/colaborativo-y-digital-el-futuro-de-la-moda/.

Martí Ventura, «Del diseño a la venta en catorce días: llega la era del 'ultrafast fashion'», Modaes Latinoamérica, 29 de mayo de 2017, https://www.modaes.com/back-stage/del-diseno-a-la-venta-en-catorce-dias-llega-la-era-delultrafast-fashion-es.html.

«El Fashion Tech Event reúne en Madrid a los principales representantes de moda y tecnología», Zona Movilidad, https://www.zonamovilidad.es/el-fashion-tech-event-reune-en-madrid-a-los-principales-representantes-de-moda-y-tecnologia.html. 